Although the celebrations were to mark the jubilee of the electron, everyone who listened to the lectures or sees the Exhibition is bound to be impressed by the still great youthfulness and vigour of the electron, for the potentialities of the new science of electronics are attracting to their study and exploitation such an amount of skill and enterprise that what has been accomplished can be regarded only as a beginning of the achievements of the liberated electron.

S. Weintroub

\section{INTERNATIONAL STANDARDS AND UNITS OF RADIOACTIVITY}

$\mathrm{T}$ HE first international radium standard was prepared by Mme. Curie in 1911 ; it consisted of $21.99 \mathrm{mgm}$. of purest radium chloride. By comparing its radiation with those from other radium sources, the latter could be measured and their radium contents expressed in grams. For the measurement of radon, grams (or cubic centimetres) of this gas are impracticable as units, and it was therefore decided to use as unit of radon the quantity which is in radioactive equilibrium with $1 \mathrm{gm}$. of radium; it was called a 'curie'. Standards for radon measurements were prepared in the form of solutions of radium salts.

Since the comparison of radioactive sources with the standards always involved measurements of ionization currents, or of the number of disintegrations per unit of time, and as especially the latter figure could be determined with much higher accuracy than the weight of the radium preparation, it became customary to base the curie directly on the number of alpha-particles emitted. According to the best measurements, $1 \mathrm{gm}$. of radium and, naturally, also the radon in equilibrium with this quantity, emits per second $3 \cdot 7^{\circ} \times 10^{10}$ alpha-particles; in recent practice, unknown radioactive sources were measured in curies by comparing their particle emission with this figure. Thus the curie became the unit in which the strength not only of members of the radium family, but also of any radioactive source, could be expressed.

This important change in the principle of radioactive measurements and in the definition of the curie was never officially sanctioned, because the International Radium Standard Commission which was responsible for the early conventions on radioactive standards and units has for a long time not been able to function satisfactorily. To remedy this situation, the proposal was made bv E. U. Condon and L. F. Curtiss (Rev. Sci. Instr., 17, 249 ; 1946) to introduce as a new unit the activity of $10^{6}$ disintegrations per second, and to call it a 'rutherford'.

It is obvious that an international agreement is imperative, whether the 'curie' in its original or in its modified form, or the new unit, the 'rutherford', should be used. The International Congress of Chemistry recently held in London offered to a number of workers in radioactivity from various countries an opportunity to discuss this question among themselves. Under the chairmanship of Prof. F. JoliotCurie (Paris), on July 19, a meeting was held by Ellen Gleditsch (Oslo), G. de Hevesy (Stockholm), Irene Joliot-Curie (Paris), C. Jacobsen (Copenhagen), W. A. Noyes, jun. (Rochester), F. A. Paneth (Durham) and G.J. Sizoo (Amsterdam); and after an exchange of views they decided to make the following recommendation.
A new committee on radioactive standards and units, comprising mainly representatives of the International Union of Chemistry and of the International Union of Physics, should be formed; but all the surviving members of the old International Radium Standard Commission should be invited to join them. As representatives of the International Union of Chemistry the names of E. Gleditsch, G. de Hevesy, Warren C. Johnson, F. Joliot-Curie, F. A. Paneth and G. T. Seaborg were suggested; as repre. sentatives of the International Union of Physics those of L. F. Curtiss, Sir Charles Darwin, R. B. Evans, C. Jacobsen, Irene Joliot-Curie and G. J. Sizoo.

Those present at the meeting were unanimously in. favour of preserving the name of 'curie' as the unit of radioactivity, but to define it now officially as the quantity of a radio-element which undergoes $3.700 \times$ $10^{10}$ radioactive transformations per second. The' final decision, however, will have to await the recommendations of an international committee on radioactive standards, the formation of which was advocated.

\section{QUANTITATIVE ASPECTS OF FREE RADICAL CHEMISTRY}

\author{
By Prof. H. W. MELVILLE, F.R.S. \\ University of Aberdeen
}

$\mathrm{T}$ HE Faraday Society held a two-day meoting in Oxford during September 23-25 to discuss "The Labile Molecule", or the quantitative aspects of free radical chemistry. The nature of the discussion seemed commonplace enough, and the tremendous activity in this field of chemistry is almost taken for granted. If we neglect the war years-and that is not quite justified - the subject seems to have grown up in about five scientific years, from a rather tentative qualitative notion to a subject of considerable precision requiring for its practice a wide variety of advanced techniques.

The attempts to unravel the mechanism of chemical reactions, the mechanism of which does not involve ions, is really the story of the development of the kinetics of homogeneous reactions. Many reactions, and especially those involving halogens, are known to involve free atoms as essential intermediates. These are, in fact, the activated 'molecules' in the old Arrhenius sense. But the idea that free organic radicals could function in a similar way, though possible in principle, came at a much later stage. The preparation of simple aliphatic radicals by the thermal decomposition of metallic alkyls, and later of a large number of simple organic molecules, provided the idea that radicals might play a part in the thermal decomposition of molecules the spontaneous decomposition of which was regarded as occurring simply by fission into simpler molecules but not radicals. Similarly, a great deal of work on the photochemical decomposition of compounds like the simpler aliphatic ketones could only be satisfactorily explained if it were assumed that the first step in the reaction consisted in the fission of a carbon - carbon bond with the production of two free radicals. Such experiments, however, gave little quantitative information about the reactivity of the radicals pro. duced, for the simple reason that the radicals mostly recombined, and therefore the velocity of decomposition was fixed by the initial rate of decomposition 
of the ketone. The kind of information that is now required about the behaviour of radicals is much more comprehensive, for it is a relatively easy matter to find whether radicals are involved in a reaction; and often the chemistry of the reaction is simple enough for the precise nature of the radical to be specified. There are at least four distinct types of radical processes about which quantitative information is required. (a) The rate of radical production by thermal activation, by thermal decomposition of relatively unstable substances such as peroxides and azo compounds and by photodecomposition of molecules. (b) The interaction of radicals with saturated molecules, in which usually an atom is removed, generating thereby another kind of radical the reactivity of which may differ markedly from that of the original radical. (c) The interaction of radicals with unsaturated molecules in which the radical adds on to the double bond, producing in this way a larger radical again of a differentactivity from that of the original. (d) The interaction of radicals with each other or with other radicals present. Such a process is the only way in which the radical is finally removed from the system.

This general list of collision types comprises the essential steps in all radical reactions, no matter whether the overall effect is the oxidation of hydrocarbons, the polymerization of vinyl compounds, the addition of carbon tetrachloride to olefins, or the addition of hydrogen bromide to double bonds between carbon atoms. It is with this more general aspect of free radical chemistry in mind that the discussion took place, and in particular the emphasis was laid on the absolute magnitude of the velocity coefficients of the above-mentioned reactions. This quantitative approach raises at once two matters. First, it is necessary to devise methods of measuring velocity coefficients, which implies a kinetic analysis of reaction mechanism with a degree of unambiguity and precision which is not easily attained. Secondly, the provision of data of this kind gives the theoretical chemist great opportunity to attempt further to correlate reactivity and chemical structure for free radical reactions.

For convenience, the discussion was broken up into the following constituent parts, each of which has contributed much to our understanding of free radical reactions : $(a)$ a theoretical part dealing with the calculation of the structure, energies and reactivity of free radicals; (b) radical reactions in the gas and liquid phases; (c) oxidation reactions; (d) polymerization reactions. Although the existence and behaviour of free radicals was first studied in the gas phase, the predominant interest appears to have shifted to the liquid phase, where the variety of effects is naturally much greater. Fortunately, it appears that the complications that might arise in working in a condensed system are not quite so serious as were first imagined.

In spite of a great deal of experimental work, the reactions of gaseous simple hydrocarbons with free radicals derived from them are still somewhat obscure, and much effort is still required before even qualitative data can be reliably obtained. For example, there is only now fairly general agreement about the energy of activation for the $\mathrm{CH}_{3}+\mathrm{H}_{2} \rightarrow$ $\mathrm{CH}_{4}+\mathrm{H}$ reaction, namely, between 7 and $9 \mathrm{k} . c a l$., the temperature-independent factor not being particularly small. Besides monoradicals, great interest is attached to the behaviour of diradicals, both in hydrocarbon chemistry and in polymerization reactions. The methylene radical can be produced either by the photodecomposition of keten or by the action of sodium vapour on methylene bromide. In the former case, the $\mathrm{CH}_{2}$ molecule attacks metallic mirrors with vigour, and it must be presumed that it acts as a diradical and not as a stable molecule $\mathrm{CH}_{2}$. Yet when produced under the conditions obtaining in a sodium 'flame', the radical does not apparently dimerize to ethylene but undergoes a variety of other reactions. Another very interesting way in which aliphatic free radicals can be formed at low temperatures is by the thermal decomposition of silver methyl and silver ethyl, in the range $-60^{\circ}$ to $-20^{\circ} \mathrm{C}$. With ethyl radicals both combination and disproportionation occur even at these low temperatures.

Oxidation of hydrocarbons to hydroperoxides is now well recognized to occur by a mechanism involving $R$ - and ROO-radicals. Even the $\mathrm{OH}$ radicals produced by the interaction of $\mathrm{Fe}^{++}$and hydrogen peroxide will provide the necessary stimulus for initiating oxidations of this type. Further, it is now becoming clear that the action of retarders in such oxidations involves the attack of the retarder by the $R O O O$ - radical. By the use of hydroquinones and similar compounds as retarders, it has been shown there is a quantitative relationship between the logarithm of the velocity coefficient and the oxidation-reduction potential of the hydroquinonequinone system. In the hydrogen-oxygen reaction, the radical corresponding to $\mathrm{RO}_{2}$ is $\mathrm{HO}_{2}$, the existence of which seems essential to the formulation of a satisfactory scheme of reaction. Similarly, a theoretical investigation confirms the stability of this radical, though no direct evidence is as yot forthcoming. Spectroscopic examination of flames provides conclusive proof of the existence of radicals, $\mathrm{BrO}$ and $\mathrm{CBr}$ being recent additions to the list. The difficulty, as always, is to determine whether such radicals exist under conditions where flame propagation is absent. The CHO radical can be identified in flame, and now its presence has been detected in photodecomposing formaldehyde by exciting it to fluorescence in the ultra-violet region of the spectrum.

The advances that have been made in studying the polymerization of vinyl compounds have provided more information about free radical processes than any other type of reaction. It is now so firmly established that these chain processes occur through the intermediary of free radicals that it almost becomes possible to use polymerization reactions as a suitable tool to study free radical reactions. Here, fortunately, there is no doubt about the general nature of the free radical, for it is either derived by the opening out of the double bond to form a diradical or by the attachment of a separately produced monoradical $R$ - to the double bond: $R+$ $\mathrm{CH}_{2}=\mathrm{CHX} \rightarrow R \cdot \mathrm{CH}_{2} \cdot \mathrm{CHX}$. One very common method of producing $R$ - radicals is from the decomposition of peroxides of the type $(R . \mathrm{COO})_{2}$, the radical $R C O O$ being first formed and carbon dioxide eliminated before $R \mathrm{COO}$ attacks the double bond. Other radical-producing substances are badly needed, and now it is reported that N-nitrosoacylarylamines will function in a similar fashion. For example, $\mathrm{N}$-nitroso acetanilide will break up with the formation of a phenyl and acetate radical and the elimination of a molecule of nitrogen. While it is of great importance to measure the rate of production of radicals in polymerization, the most important step is the interaction of the radical derived from the 
monomer, namely, $R-+\mathrm{CH}_{2}-\mathrm{CH} X$ with another monomer molecule to form a still larger radical-the all-important growth-step.

In order to compute the velocity coefficient from the rate at which monomer disappears, it is necessary to know the stationary concentration of the free radicals. Fortunately, this can now be done, since we know the rate of production of radicals and the time required for the concentration to fall to half its value when the source of activation is removed, either by the use of intermittent radiation when the life is short, or by direct observation of the decay of the rate of polymerization when the life is long. Unfortunately, the results are confined to vinyl acetate, methyl methacrylate and styrene at present; hence it is a little too early to see how structure affects reactivity. In the meantime, information has been obtained in a somewhat less direct manner. By studying the copolymerization of two monomers $A$ and $B$, and analysing the composition of the co. polymer formed during the reaction, starting with known concentrations of $A$ and of $B$, it is possible to calculate the value of two factors, namely, the ratio of the rates of reaction of $A$ and of $B$ with a $B$-radical, and the ratio of the rates of reaction of $A$ and of $B$ with an $A$-radical. Therefore, $A$ may be kept fixed and the nature of $B$ altered by changing the nature of the substituent $X$ of the vinyl compound $\mathrm{CH}_{2}=\mathrm{CHX}$. In this way it is possible to get a whole series of monomer reactivity ratios which give relative values for the growth steps in polymerization.

This material has provided the data for tentative theories of reactivity. As a first approximation, it may be supposed that there can be ascribed a potential reactivity of the free radical and also of the monomer which is going to be attacked. It has been suggested that the magnitude of the electric change on the group $X$ will play a predominant part in effecting radical - molecule interaction, and there is some evidence to support almost a purely electrostatic approach to the theory. On the other hand, there is another school of thought which argues in rather a different way; basing relative reactivity on the relative values of the resonance energy of the radical-monomer system before, during and after interaction. Qualitatively, it is possible to go some way with such theories, and most important of all, to incorporate radical-monomer reactivity in the more general theory of the effect of the character of substituents in the reactivity of neighbouring groups. There is, however, a long way to go before any attempt is made to compute the absolute value of velocity coefficients. Indeed, it is only fair to say that in nearly all cases the temperature-independent and temperature-dependent factors have not yet been separately determined, and the theory is wholly based on the values of velocity coefficients determined at one temperature.

When polymerization is carried out in solution, it often happens that the polymer radicals attack the solvent molecule, removing an atom, regenerating another radical which reacts with further molecules of monomer. Thus the kinetic chain is continued, but the continuity of the polymor chain is broken in this so-called transfer reaction. By observing the influence of solvent on the degree of polymerization of the polymer, it is possible to compare the relative activities of the radical attacking the monomer on one hand and the solvent on the other. Absolute values of the radical-solvent interaction can then at once be obtained if the growth coefficient is known. Polymer reactions thereby provide a very conveniont and sensitive means of studying a great variety of reactions. For example, when the solvent is hydrocarbon the dominant factor that seems to govern the reactivity is the bond-strength of the weakest $\mathrm{C}-\mathrm{H}$ bond. Further, if the hydrocarbon radical so produced is stabilized by possessing a large resonance energy, it may not react with the monomer, and so the kinetic chain comes to a stop. The solvent then acts as a retarder for polymerization and the molecular woight of the polymer is cut down. There is therefore little formal difference between transfer and retardation.

Degradation of vinyl polymers also presents interesting information on radical reactivity. In some cases it would appear that the mechanism of degradation is really like polymerization in reverse. If it should happen that thermal fission of a polymer occurs into two free radicals-a relatively difficult process because $\mathrm{C}-\mathrm{C}$ bonds have to be broken-these radicals are rather unstable in the sense that not much energy, for example, $16 \mathrm{k} . c a l .$, is required to remove monomer units. Such radicals are therefore very easily removed from the system. Like the building up of polymers by chain processes, this degradation can likewise be materially diminished by adding substances that will stabilize the radicals and prevent further degradation.

\section{PLANT TUMOURS AND ANIMAL CANCER}

\author{
By R. S. de ROPP \\ New York Botanical Garden
}

$\mathrm{T}$ HE discovery by Smith and Townsend ${ }^{1}$ that crown-gall disease of plants was caused by a bacterium (Phytomonas tumefaciens) aroused considerable interest among students of cancer. The view that animal cancer was of bacterial origin was vigorously put forward by Smith, who published several papers emphasizing the resemblance of plant galls produced by $P h$. tumefaciens and to cancerous neoplasms. He distinguished several different types of plant tumours which he compared to sareomas, carcinomas and teratomas. Secondary tumours which sometimes formed on infected plants at points remote from the original tumours were compared by Smith to the metastases observed in animal cancer. Smith was convinced that the galls produced by $P h$. tumefaciens always contained the bacteria within their cells. As investigators of animal cancer failed to isolate any bacterium which could be definitely associated with the formation of cancer, interest in Smith's views tended to decrease.

In 1918 a paper was published by C. O. Jensen ${ }^{2}$ which gave the first indications that tumours initiated by $P h$.tumefaciens might continue to grow as tumours in the absence of the bacteria. Jensen worked with mangolds and sugar beets and succeeded in grafting tumour tissue from these plants on to normal roots. Many of these grafts took, and the tumour tissue continued to grow as a tumour. From all such transplanted tumours Jensen found that the original crown-gall bacteria had disappeared.

The importance of these findings was overlooked until Braun and White ${ }^{3}$ began their studies on the 